\title{
含醛基及叠氮基氮杂糖中间体的合成
}

\author{
李小六* 魏思楠张宏波张平竹王玮陈 华* \\ (河北大学化学与环境科学学院 河北省化学生物学重点实验室 保定 071002)
}

\begin{abstract}
摘要 以 $D$-甘露糖为原料, 分别利用芐胺双取代和 aza-Wittig 反应，再经氧化和亲核取代反应，合成了带保护基的含叠 氮基及醛基的五元及六元氮杂糖中间体，对相关反应条件、基团保护及反应机理等进行了探讨，并提供了一种选择性 脱除三苯甲基保护基的方法.
\end{abstract}

关键词＼cjkstart氮杂糖; 芐胺双取代; aza-Wittig 反应; 三苯甲基; 选择性去保护

\section{Syntheses of Azasugar Intermediates Containing Aldehyde Group or Azide Group}

\author{
Li, Xiaoliu* Wei, Sinan Zhang, Hongbo Zhang, Pingzhu Wang, Wei Chen, Hua* \\ (Key Laboratory of Chemical Biology of Hebei Province, College of Chemistry and Environmental Science, \\ Hebei University, Baoding 071002)
}

\begin{abstract}
Protected five and six membered azasugar intermediates containing aldehyde group or azide group were synthesized using $D$-mannose as starting material via benzyl amine disubstitution or aza-Wittig reaction, then the oxidation and $\mathrm{S}_{\mathrm{N}} 2$ substitution reactions, respectively. The studies on the reaction conditions, the protecting groups and the reaction mechanism were also carried out, providing a protocol to remove trityl (Tr) group selectively.

Keywords azasugar; benzylamine disubstitution; aza-Wittig reaction; trityl; selective deprotection
\end{abstract}

氮杂糖(azasugar)是糖环上氧原子被氮取代的糖类 衍生物, 具有抗糖尿病、抗癌、抗病毒(艾滋病)感染以 及抗免疫系统紊乱等活性 ${ }^{[1 \sim 4]}$. 作为一类非常重要的糖 苷酶抑制剂 ${ }^{[5,6]}$, 氮杂糖药物 Glyset 和 Zavesca (米格列 醇和 $N$-丁基脱氧野尻霉素, 图 1)已分别用于治疗血糖 升高引起的 II 型糖尿病及糖苷酶紊乱导致的 Gaucher 病 ${ }^{[3,7,8]}$. 此外, 一些含氮杂糖的复杂衍生物也具有良好 的生物活性, 如氮杂糖类核苷化合物 (ImmH1) 能够有效 地抑制 $\mathrm{T}$ 淋巴细胞的增殖 ${ }^{[9]}$, 连有香豆素的氮杂糖 $(\mathbf{A})^{[10]}$ 以及氮杂二糖 $(\mathbf{B})^{[11]}$ 具有良好的糖苷酶抑制活性 (图 1). 因此, 氮杂糖以其重要的生理活性及良好的药用 价值受到广泛关注. 目前, 简单氮杂糖的合成方法较为 成熟多样 ${ }^{[12]}$, 但由于反应路线普遍较长, 且操作繁琐, 极大的限制了其复杂衍生物的进一步研究. 为了能更好 的拓展氮杂糖的应用, 在氮杂糖分子中引入含适于后续
反应的官能团，如醛基、叠氮基(氨基)等，对于合成和发 现具有良好生物活性的复杂氮杂糖衍生物具有重要的 意义, 如图 1 中化合物 $\mathbf{A}, \mathbf{B}$ 和 $\mathrm{ImmH} 1$ 均是由带有特定 反应基团的氮杂糖中间体制备. 最近，实验室利用糖 醛、胺或糖基叠氮和颈基乙酸的一锅法环合反应合成了 一类具有良好免疫调节活性的含噻唑烷-4-酮的类核苷 和假双糖衍生物(化合物 $\mathbf{C}$ 和 $\mathbf{D}$, 图 1) 1 $^{[13,14]}$, 鉴于氮杂糖 在免疫调节中的良好作用 ${ }^{[15]}$, 实验室拟进一步合成其 氮杂糖苷衍生物(化合物 $\mathbf{E}$ 和 $\mathbf{F}$ ，图 1)，以探讨和发现新 型氮杂糖类免疫调节剂. 因此, 在实验室已有糖化学研 究的基础上 ${ }^{[16,17]}$, 并出于为课题组后续合成研究提供原 料为目的，本文分别利用茮胺双取代 ${ }^{[18,19]}$ 和 aza-Wittig 反应 ${ }^{[20,21]}$, 设计合成了带有保护基的分别含醛基和叠氮 基的五元(Scheme 1)、六元氮杂糖中间体(Schemes 2,3). 对相关反应条件、基团保护及反应机理等进行了探讨,

*E-mail: lixl@hbu.cn, hua-todd@hbu.cn

Received March 26, 2012; revised May 9, 2012; published online May 16, 2012.

Project supported by the National Natural Science Foundation of China (Nos. 20972039, 21172051), the Medicinal Joint Funds of the Natural Science Foundation of Hebei Province and the Shijiazhuang Pharmaceuitical Group (CSPC) Foundation (Nos. B2011201169, B2012201113), and the Natural Science Foundations of Education Department of Hebei Province (Nos. ZH2011110, Y20111119).

国家自然科学基金(Nos. 20972039, 21172051)、河北省自然科学基金石药集团医药联合基金(Nos. B2011201169, B2012201113)、河北省教育厅自然科 学基金(Nos. ZH2011110, Y2011119)资助项目. 
可为将来类似化合物的合成提供有益的参考.<smiles>CCCCN1C[C@H](O)[C@@H](O)[C@H](O)C1CO</smiles>

$N$-butyl-1-deoxynojirimycin<smiles>Nc1ncnc2c(C3N[C@H](CO)[C@@H](O)[C@H]3O)c[nH]c12</smiles><smiles>CCNc1ccc2c(c1)OC(=O)C(C(=O)NC[C@H]1NC(CO)[C@H](O)C1O)C2</smiles>

A<smiles>COC1[C@H](O)C(CO)O[C@H]1C1SCC(=O)N1[N+](=O)[O-]</smiles><smiles>COC1OC([C@@H]2SCC(=O)N2CC2O[C@H](OC)[C@@H](O)[C@H]2O)[C@@H](O)[C@@H]1O</smiles>

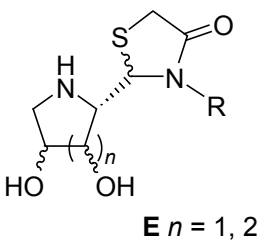

$\mathbf{E} n=1,2$<smiles>O=C1CS[C@@H](C2NC[C@@H](O)[C@H]2O)N1CC1NCC[R9]1(O)O</smiles>

图 1 具有生物活性的氮杂糖及其衍生物

Figure 1 Some bioactive azasugars and their derivatives

\section{1 结果与讨论}

\section{1 含醛基及叠氮基五元氮杂糖中间体的合成}

五元氮杂糖的合成已有较系统的研究 ${ }^{[4,12,22]}$, 本文 以 $D$-甘露糖为起始原料, 通过经典的苠胺双取代反应 合成了具有五元氮杂糖框架的中间体 $\mathbf{1}^{[18,19]}$. 后续的含 醛基及叠氮基五元氮杂糖中间体的合成路线如 Scheme 1 所示. 理论上, 化合物 1 经异亚丙基脱除和高碘酸钠 $\left(\mathrm{NaIO}_{4}\right)$ 氧化即可直接获得 $\mathrm{N}$ 原子苠基 $(\mathrm{Bn})$ 保护的含醛 基的五元氮杂糖中间体. 但考虑到苠基脱除时, 硫原子 的存在会导致钯碳 $(\mathrm{Pd} / \mathrm{C})$ 中毒而影响课题组后续的实验 (以此醛为原料合成噻唑烷酮糖苷衍生物), 因此在氧化 邻二醇之前, 用可在酸条件下脱除且对氨基选择性好的 叔丁氧羰基(Boc)替换苠基作为 $\mathrm{N}$ 原子上的保护基.

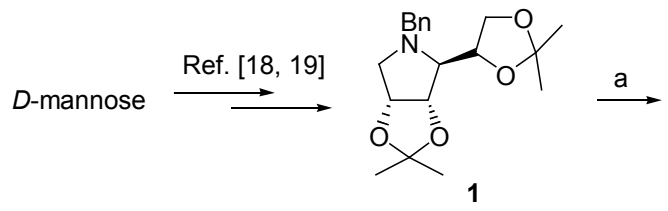

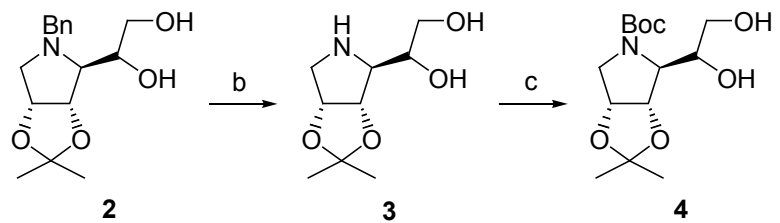

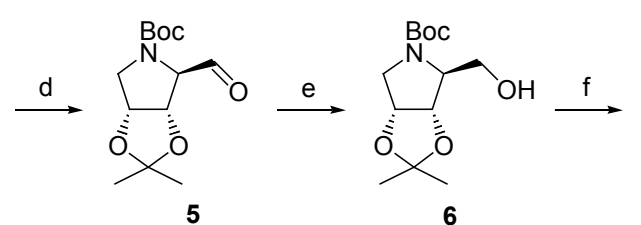

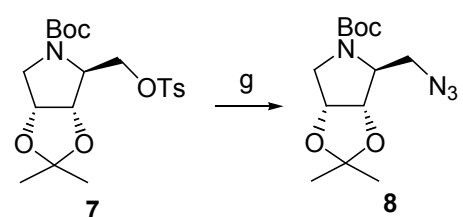

Reagents and conditions: (a) $80 \% \mathrm{AcOH}, 60{ }^{\circ} \mathrm{C}$; (b) $\mathrm{H}_{2}, 10 \% \mathrm{Pd} / \mathrm{C}$; (c) $\mathrm{Boc}_{2} \mathrm{O}, \mathrm{MeOH}, \mathrm{Et}_{3} \mathrm{~N}, 51 \%$, 3 steps; (d) $\mathrm{NalO}_{4}, 80 \% \mathrm{EtOH}, 92 \%$; (e) $\mathrm{NaBH}_{4}, 80 \% \mathrm{EtOH}, \mathrm{H}_{2} \mathrm{O}, 94 \%$; (f) $\mathrm{TsCl}, \mathrm{Py}, 0{ }^{\circ} \mathrm{C}, 92 \%$; (g) $\mathrm{NaN}_{3}$, DMF, $100{ }^{\circ} \mathrm{C}, 94 \%$

\section{Scheme 1}

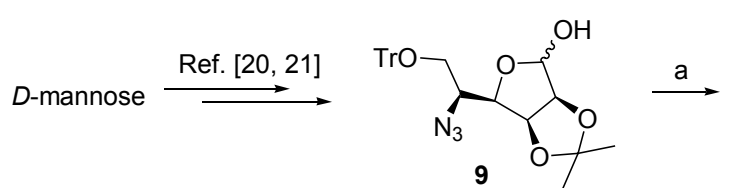<smiles>CC1(C)OC2CN[C@H](CO[Ga])[C@H](O)C2O1</smiles>

10

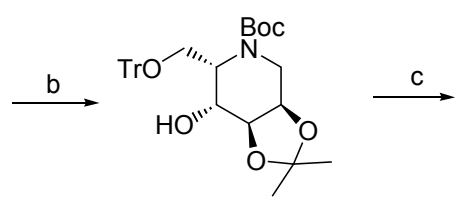

11

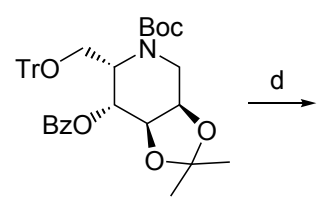

12

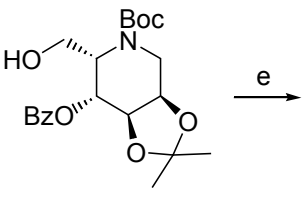

13<smiles>CC1(C)OC2CN(C(=O)OCc3ccccc3)[C@H](C=O)[C@H]2O1</smiles>

14

Reagents and conditions: (a) i) $\mathrm{Ph}_{3} \mathrm{P}, \mathrm{THF}, 70{ }^{\circ} \mathrm{C}$; ii) $\mathrm{NaBH}_{4}, \mathrm{MeOH}$, $85 \%$; (b) $\mathrm{Boc}_{2} \mathrm{O}, \mathrm{Et}_{3} \mathrm{~N}, \mathrm{MeOH}, 80 \%$; (c) $\mathrm{BzCl}$, pyridine, $0{ }^{\circ} \mathrm{C} \sim$ r.t., $90 \%$; (d) $\mathrm{FeCl}_{3} \cdot 6 \mathrm{H}_{2} \mathrm{O}, \mathrm{DCM}, 0{ }^{\circ} \mathrm{C}, 72 \%$; (e) Dess-Martin periodinane, DCM, $90 \%$

\section{Scheme 2}


<smiles>CCOC[C@H]1NCC2OC(C)(C)OC2[C@H]1O</smiles>

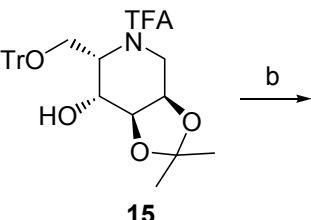<smiles>CN1CC2OC(C)(C)[C@@H](CO)[C@@H](O)C2OC1(C)C</smiles>
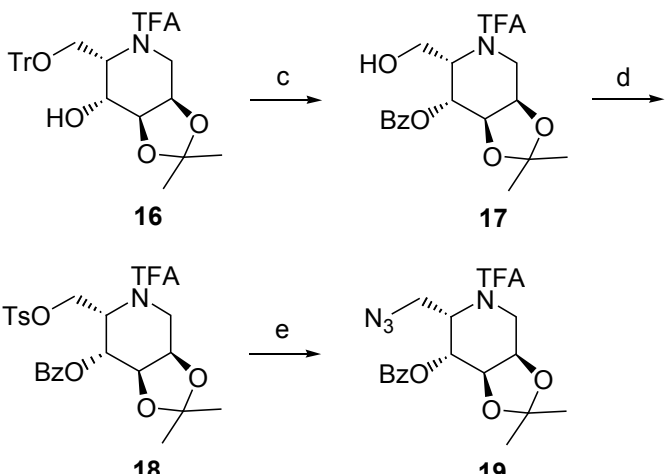

19

Reagents and conditions: (a) TFAA, Py, DCM, $-40{ }^{\circ} \mathrm{C}, 80 \%$; (b) BzCl, pyridine, $0{ }^{\circ} \mathrm{C} \sim$ r.t., $90 \%$; (c) $\mathrm{FeCl}_{3} \cdot 6 \mathrm{H}_{2} \mathrm{O}, \mathrm{DCM}, 0{ }^{\circ} \mathrm{C}, 90 \%$; (d) $\mathrm{TsCl}$, $\mathrm{Et}_{3} \mathrm{~N}, \mathrm{DCM}, 0{ }^{\circ} \mathrm{C} \sim$ r.t; (e) $\mathrm{NaN}_{3}$, DMF, $100{ }^{\circ} \mathrm{C}, 75 \%$, 2 steps

\section{Scheme 3}

Boc 保护反应的条件一般为质子性溶剂, 加碱作为缚酸 剂. 合成过程中分别尝试了 $\mathrm{NaOH}$ 和 $\mathrm{Et}_{3} \mathrm{~N}$ 的碱性条件, 结果在 $\mathrm{NaOH}$ 条件下, 反应时间短但产率低, 而在 $\mathrm{Et}_{3} \mathrm{~N}$ 体系下, 以 $80 \%$ 的高产率得到化合物 4 . 进而以高碘酸 钠 $\left(\mathrm{NaIO}_{4}\right)$ 为氧化剂, $80 \%$ 的乙醇水溶液中, 以 $92 \%$ 的产 率氧化化合物 4 中的邻二醇为醛基, 得到化合物 $\mathbf{5}$. 再 经嗍氢化钠 $\left(\mathrm{NaBH}_{4}\right)$ 还原、对甲苯磺酰基( $\left.\mathrm{Ts}\right)$ 化, 及与叠 氮化钠 $\left(\mathrm{NaN}_{3}\right)$ 的 $\mathrm{S}_{\mathrm{N}} 2$ 亲核取代反应, 高产率的合成了含 叠氮基的氮杂糖中间体 8 .

\section{2 含醛基及叠氮基六元氮杂糖中间体的合成}

\subsection{1 含醛基的六元氮杂糖中间体的合成}

含醛基的六元氮杂糖中间体的合成路线如 Scheme 2 所示. 六元氮杂糖骨架的构建主要通过分子内 azaWittig 反应关环形成亚胺(图 2), 再经还原得到 ${ }^{[20,21]}$. 由 于在 aza-Wittig 反应过程中以四氢呋喃(THF)为溶剂, 因 此先后尝试了直接在 THF 溶剂中以硼氢化钠 $\left(\mathrm{NaBH}_{4}\right)$ 或 氯基硼氢化钠 $\left(\mathrm{NaBH}_{3} \mathrm{CN}\right)$ 进行还原, 但反应效果不佳 (产率 $40 \%$ ). 于是在 9 形成氮关环产物后, 将 $\mathrm{THF}$ 减压 蒸除, 改甲醇为反应溶剂, 以 $\mathrm{NaBH}_{4}$ 为还原剂, 以较高 的产率(75\%)得到六元氮杂糖化合物 $\mathbf{1 0}$.

为了获得到含醛基的六元氮杂糖中间体，首先将化 合物 10 中的亚胺基和羟基分别用 Boc 和苯甲酰基(Bz) 进行保护得到中间体 12, 然后尝试将原有的三苯甲基 (Tr)脱除以裸露羟甲基用于氧化. 由于 Boc, $\operatorname{Tr}$ 以及异亚 丙基均在酸性条件下易脱除, 实验中对选择性脱除三苯 甲基的反应条件进行了详细探讨. 先后尝试了不同浓度 的醋酸水溶液 $(80 \%, 50 \%$ 和 $30 \%$ 的 $\mathrm{AcOH})$ 、三氟醋酸水
溶

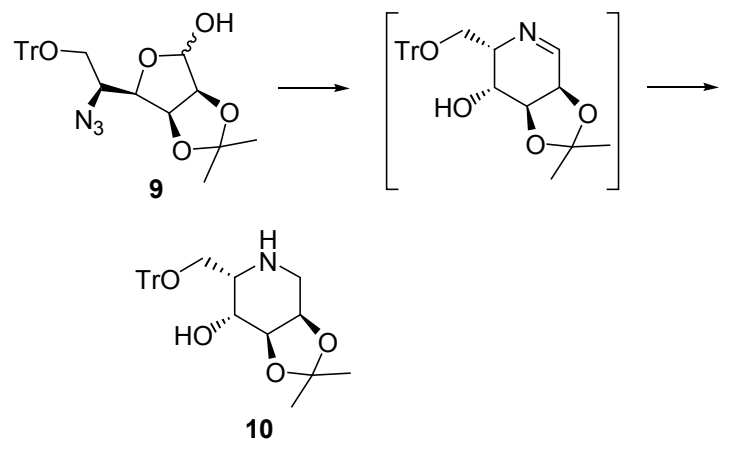

图 2 aza-Wittig 反应

Figure 2 aza-Wittig Reaction

液( $60 \%, 50 \%$ 和 $30 \%$ 的 $\mathrm{CF}_{3} \mathrm{COOH}$ ) 和盐酸水溶液 $(2,1$ 和 $0.5 \mathrm{~mol} \cdot \mathrm{L}^{-1}$ 的 $\mathrm{HCl}$ ), 希望可以通过调整反应体系酸度和 反应温度实现选择性脱除三苯甲基，但均未能得到预期 结果. 后改用在三氟化嗍 $\left(\mathrm{BF}_{3} \cdot \mathrm{Et}_{2} \mathrm{O}\right)$ 、三氯化铁 $\left(\mathrm{FeCl}_{3} \cdot 6 \mathrm{H}_{2} \mathrm{O}\right)$ 等路易斯酸条件下进行反应，最终发现 $\mathrm{FeCl}_{3} \cdot 6 \mathrm{H}_{2} \mathrm{O}$ 在二氯甲烷 $(\mathrm{DCM})$ 中 $0{ }^{\circ} \mathrm{C}$ 条件下, 可以选择 性脱除三苯甲基得到目标化合物 13. 进行该反应时，当 原料 12 投料量小于 $100 \mathrm{mg}$ 时，产率可达 $80 \%$ 以上. 但 是放大量至 $1 \mathrm{~g}$ 时，产率不足 $30 \%$. 我们进一步以原料 12 为 $1 \mathrm{~g}$, 粉末状 $\mathrm{FeCl}_{3} \cdot 6 \mathrm{H}_{2} \mathrm{O}$ 为 $0.6 \mathrm{~g}$ (相当于 12 的 1.5 equiv.)进行反应为例, 重点探讨了反应时间和溶剂用量 (反应物浓度)对反应产率的影响. 结果表明，在反应初 始时, 随着反应时间的延长, 产物明显增加. 但 $20 \mathrm{~min}$ 后, 虽仍有原料 12 末反应完全, 但产物逐渐减少, 并出 现难于薄层展开的大极性黑色物质. 推测有可能随着反 应时间的延长, 有异亚丙基脱除等副产物生成, 导致 $\mathrm{FeCl}_{3}$ 与羟基络合形成配合物，从而使产物减少。反应 体系温度越高这种现象越明显. 此外, 随着反应体系中 二氯甲烷溶剂的增多，原料 $\mathrm{FeCl}_{3}$ 可以尽可能多的溶解 并参与反应. 但当溶剂增加到 $200 \mathrm{~mL}$ 甚至更大时, 反 应趋于平衡. 因此, 最终选择 $1 \mathrm{~g}$ 原料 $\mathbf{1 2}, 0.6 \mathrm{~g}$ $\mathrm{FeCl}_{3} \cdot 6 \mathrm{H}_{2} \mathrm{O}$ 溶解在 $200 \mathrm{~mL}$ 二氯甲烷中, $0{ }^{\circ} \mathrm{C}$ 条件下, 反 应 $20 \mathrm{~min}$, 以 $72 \%$ 的反应产率得到目标化合物 13. 未反 应完的原料 12 通过简单柱层析分离后，可以回收重复 使用. 该条件也适于进一步的放大实验. 在得到纯净的 化合物 13 后，以 Dess-Martin 试剂为氧化剂, $90 \%$ 的反应 产率得到化合物 $\mathbf{1 4}$.

\subsection{2 含叠氮基的六元氮杂糖中间体的合成}

参照已有的含叠氮基的五元氮杂糖 $\mathbf{8}$ 的制备方法, 计划在 13 的伯羟基上引入离去基团, 通过取代反应合 成含叠氮基的六元氮杂糖 19. 但是在 13 引入离去基团 后，化合物不稳定，直接分子内并环得到了氮杂糖并噁 
唑衍生物 13-1, 反应机理如 Scheme 4 所示 ${ }^{[23]}$. 此反应在 合成含叠氮基的五元氮杂糖中间体 $\mathbf{8}$ 时并未发现，分析 可能是由于双五元并环结构张力较大, 从而较难形成. 因此, 改用三氟乙酰基(TFA)对 $\mathrm{N}$ 原子保护, 并成功地 合成了含叠氮基的六元氮杂糖的中间体 19.

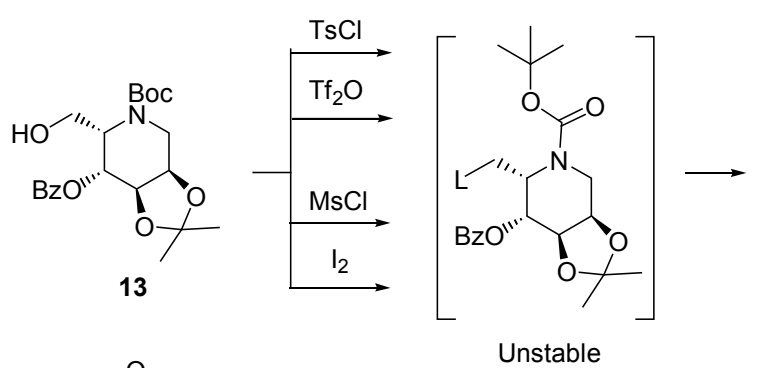<smiles>CC(C)(C)O[C@H]1C2OC(C)(C)OC2CN2C(=O)OCC12</smiles>

Unstable

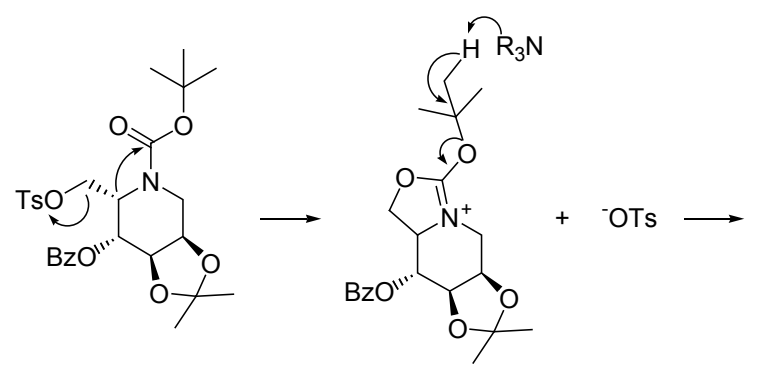

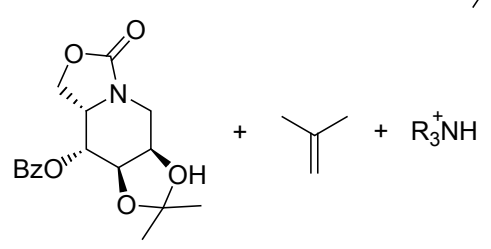

Scheme 4

产物及中间体的结构由核磁共振 $\left({ }^{1} \mathrm{H}\right.$ 和 $\left.{ }^{13} \mathrm{C} \mathrm{NMR}\right)$ 及高分辨质谱(HR-ESI-MS)等分析方法确定. 值得注意 的是, 在 Boc 保护的五、六元氮杂糖衍生物的核磁谱图 上均可以明显的观察到立体异构现象. 这可能是由于氮 上的 Boc 基团与孤对电子不能自由翻转, 形成了类似于 几何异构的旋转异构体(Rotamers)所致, 这与我们之前 的发现 ${ }^{[16]}$ 以及文献[24]报道一致.

\section{2 结论}

综上所述, 以 $D$-甘露糖为起始原料, 经 8 步和 11 步, 以 $25 \%$ 和 $21 \%$ 的总产率分别合成了含醛基和叠氮基 的五元氮杂糖中间体 5 和 8; 经 12 步和 13 步, 以 $21 \%$ 和 $13 \%$ 的总产率分别合成了含醛基和叠氮基的六元氮 杂糖中间体 14 和 19. 为进一步合成含功能基的复杂氮

杂糖衍生物提供原料. 此外, 在 $\mathrm{FeCl}_{3}$ 路易斯酸的作用 下，可以有效的选择性脱除三苯甲基保护基，提供了一 种糖环羟基选择性保护和去保护的方法.

\section{3 实验部分}

\section{1 仪器与试剂}

熔点由 $\mathrm{SGW}^{\circledR} \mathrm{X}-4$ 显微熔点仪(温度计未校正)测 定; 旋光由 $\mathrm{SGW}^{\circledR}$ X-1 自动旋光仪测定; 核磁共振谱用 BRUKER AC-P600 (600 MHz)型核磁共振仪测定, TMS 为内标; 高分辨质谱(ESI)用 FTICR-MS (Ionspec 7.0T) 型质谱仪测定; 层析用硅胶(200 300 目，300 400 目) 为青岛海洋化工厂产品. 本文所用其他试剂均为分析 纯, 无水试剂均按常规方法处理, 水为二次蒸馏水.

\section{2 五元氮杂糖醛基及叠氮基中间体的合成}

3.2.1 (R)-1-[(3aS,4S,6aR)-2,2-二甲基-5-芐基四氢$3 a H-[1,3]$ 二氧戊烷 $[4,5-c]$ 吡咯-4-基]-1,2-乙二醇 $(2)$ 的 合成

以 $D$-甘露糖为其始原料, 参考文献 $[18,19]$ 的方法, 利用茮胺双取代反应合成氮杂糖中间体 (3aS,4S,6aR)-2,2-二甲基-5-苠基-4-[(R)-2,2-二甲基-1,3二氧戊烷-4-基]四氢 $-3 a H-[1,3]$ 二氧戊烷 $[4,5-c]$ 吡咯(1).

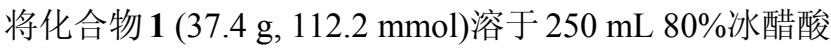
水溶液中, 油浴 $50{ }^{\circ} \mathrm{C}$ 反应 $30 \mathrm{~h}$, 加饱和 $\mathrm{NaOH}$ 溶液中 和至碱性, 冷却至室温后, 用 $\mathrm{CH}_{2} \mathrm{Cl}_{2}$ 萃取 $(200 \mathrm{~mL} \times 3)$, 饱和食盐水洗 $(100 \mathrm{~mL} \times 2)$, 无水硫酸钠 $\left(\mathrm{NaSO}_{4}\right)$ 干燥有 机相, 抽滤, 减压浓缩, 硅胶柱层析 $[V$ (石油醚) : $V($ 乙酸 乙酯 $)=5: 1$ 分离得褐色油状物, 产率 $80 \%$. $[\alpha]_{\mathrm{D}}^{25}+$ 13.3 (c 1.0, $\mathrm{CHCl}_{3}$ ); ${ }^{1} \mathrm{H} \mathrm{NMR}\left(600 \mathrm{MHz}, \mathrm{CDCl}_{3}\right) \delta: 1.36$ (s, 3H, $\left.\mathrm{CH}_{3}\right), 1.64\left(\mathrm{~s}, 3 \mathrm{H}, \mathrm{CH}_{3}\right), 3.09$ (d, J=4.2 Hz, 2H, $\left.\mathrm{CH}_{2}\right), 3.36(\mathrm{~d}, J=6.6 \mathrm{~Hz}, 1 \mathrm{H}, \mathrm{CH}), 3.43 \sim 3.49(\mathrm{~m}, 1 \mathrm{H}$, $\mathrm{CH}), 3.54 \sim 3.72\left(\mathrm{~m}, 2 \mathrm{H}, \mathrm{CH}_{2}\right), 4.11,4.17(\mathrm{~d}, J=12.6 \mathrm{~Hz}$, $\left.2 \mathrm{H}, \mathrm{PhCH}_{2}\right), 4.68(\mathrm{~d}, J=6.0 \mathrm{~Hz}, 1 \mathrm{H}, \mathrm{CH}), 4.81 \sim 4.86(\mathrm{~m}$, $1 \mathrm{H}, \mathrm{CH}), 7.28 \sim 7.36(\mathrm{~m}, 5 \mathrm{H}, \mathrm{ArH}) ;{ }^{13} \mathrm{C} \mathrm{NMR}(125 \mathrm{MHz}$, $\left.\mathrm{CDCl}_{3}\right) \delta: 23.5,26.7,57.9,61.9,64.3,69.2,71.9,82.7$, 84.4, 111.7, 127.5, 128.6, 129.3, 139.0; HRMS (ESI) calcd for $\mathrm{C}_{16} \mathrm{H}_{23} \mathrm{NO}_{4} \mathrm{Na} 316.1525$, found 316.1528.

$3.2 .2(R)-1-[(3 a S, 4 S, 6 a R)-2,2-$ 二甲基四氢-3aH-[1,3]二 氧戊烷 $[4,5-c]$ 吡咯-4-基]-1,2-乙二醇(3)的合成

将化合物 $2(5.0 \mathrm{~g}, 17.0 \mathrm{mmol})$ 溶于无水乙醇, 加入 冰醋酸 $1.5 \mathrm{ml}, 10 \%$ 钯碳 $(\mathrm{Pd} / \mathrm{C}) 1.0 \mathrm{~g}$, 抽真空后通入氢 气, 剧烈搅拌下反应 $40 \mathrm{~h}$, 加入碳酸钠中和, 抽滤回收 钯碳, 减压浓缩, 硅胶柱层析 $[V$ (石油醚) : $V($ 乙酸乙 酯 $)=3: 1$ 分离得浅黄色固体 3, 产率 81\%. m.p. 73.0 $74.0{ }^{\circ} \mathrm{C} ;[\alpha]_{\mathrm{D}}^{25}+3.5\left(c 1.0, \mathrm{CHCl}_{3}\right) ;{ }^{1} \mathrm{H}$ NMR $(600 \mathrm{MHz}$, $\left.\mathrm{CDCl}_{3}\right) \delta: 1.34\left(\mathrm{~s}, 3 \mathrm{H}, \mathrm{CH}_{3}\right), 1.51\left(\mathrm{~s}, 3 \mathrm{H}, \mathrm{CH}_{3}\right), 3.24 \sim 3.38$ 
$\left(\mathrm{m}, 2 \mathrm{H}, \mathrm{CH}_{2}\right), 3.56(\mathrm{~d}, J=5.4 \mathrm{~Hz}, 1 \mathrm{H}, \mathrm{CH}), 3.67 \sim 3.75$ (m, 3H, $\left.\mathrm{CH}_{2}, \mathrm{CH}\right), 4.78$ (dd, $\left.J=6.0,1.2 \mathrm{~Hz}, 1 \mathrm{H}, \mathrm{CH}\right), 4.88$ $(\mathrm{t}, J=4.8 \mathrm{~Hz}, 1 \mathrm{H}, \mathrm{CH}) ;{ }^{13} \mathrm{C} \mathrm{NMR}\left(125 \mathrm{MHz}, \mathrm{CDCl}_{3}\right) \delta$ : 23.0, 25.2, 51.4, 63.5, 66.6, 69.2, 80.0, 82.8, 111.7; HRMS (ESI) calcd for $\mathrm{C}_{9} \mathrm{H}_{18} \mathrm{NO}_{4}$ 204.1236, found 204.1245.

3.2.3 (3aS,4S,6aR)-叔丁基-2,2-二甲基-4-[(R)-1,2-二 差基乙基] 二氢 $-3 a H-[1,3]$ 二氧戊烷 $[4,5-c]$ 吡咯-5(4H)羧酸酯(4)的合成

将化合物 3 (2.8 g, $13.7 \mathrm{mmol}$ ) 溶于 $30 \mathrm{~mL}$ 甲醇中, 加入 $10 \mathrm{~mL}$ 三乙胺 $\left(\mathrm{Et}_{3} \mathrm{~N}\right)$, 二碳酸二叔丁酯 $\left(\mathrm{Boc}_{2} \mathrm{O}, 6.1\right.$ $\mathrm{g}, 27.4 \mathrm{mmol}$ ), 室温搅拌 $1 \mathrm{~h}$, 反应完全后用 $\mathrm{CH}_{2} \mathrm{Cl}_{2}$ 萃取 $(200 \mathrm{~mL} \times 3)$, 饱和食盐水洗 $(100 \mathrm{~mL} \times 2)$, 无水 $\mathrm{NaSO}_{4}$ 干燥有机相, 抽滤, 减压浓缩, 硅胶柱层析 $[V($ 石油 醚)： $V$ (乙酸乙酯 $)=6 ： 1$ ] 分离得白色固体 4, 产率 $80 \%$. m.p. $85.6 \sim 87.4{ }^{\circ} \mathrm{C} ;[\alpha]_{\mathrm{D}}^{25}+53.9$ (c 1.0, $\left.\mathrm{CHCl}_{3}\right) ;{ }^{1} \mathrm{H}$ NMR (600 MHz, $\left.\mathrm{CDCl}_{3}\right) \delta: 1.32\left(\mathrm{~d}, J=4.2 \mathrm{~Hz}, 3 \mathrm{H}, \mathrm{CH}_{3}\right)$, $1.40\left(\mathrm{~d}, J=6.6 \mathrm{~Hz}, 3 \mathrm{H}, \mathrm{CH}_{3}\right), 1.49\left(\mathrm{~s}, 9 \mathrm{H}, 3 \mathrm{CH}_{3}\right), 3.41 \sim$ $4.51\left(\mathrm{~m}, 2 \mathrm{H}, \mathrm{CH}_{2}\right), 3.55 \sim 3.63(\mathrm{~m}, 1 \mathrm{H}, \mathrm{CH}), 3.73 \sim 3.83$ $\left(\mathrm{m}, 2 \mathrm{H}, \mathrm{CH}_{2}\right), 4.10 \sim 4.17(\mathrm{~m}, 1 \mathrm{H}, \mathrm{CH}), 4.76 \sim 4.78(\mathrm{~m}$, $1 \mathrm{H}, \mathrm{CH}) ;{ }^{13} \mathrm{C} \mathrm{NMR}\left(125 \mathrm{MHz}, \mathrm{CDCl}_{3}\right) \delta: 23.6,23.6,25.9$, $25.9,27.3,53.5,54.2,62.9,63.6,64.7,65.7,73.1,73.4$, $79.5,80.1,83.1,83.8,110.9,110.9,155.4,156.2$; HRMS (ESI) calcd for $\mathrm{C}_{14} \mathrm{H}_{26} \mathrm{NO}_{6} 304.1760$, found 304.1754.

$3.2 .4(3 a S, 4 R, 6 a R)$-叔丁基-2,2-二甲基-4-甲酰基二 氢-3aH-[1,3] 二氧戊烷 $[4,5-c]$ 吡咯- $5(4 H)$-羒酸酯 $(5)$ 的 合成

将化合物 4 (3.3 g, $10.9 \mathrm{mmol})$ 溶于 $50 \mathrm{~mL} 80 \%$ 的乙 醇水溶液，冰浴下加入高碘酸钠 $\left(\mathrm{NaIO}_{4}, 4.7 \mathrm{~g}, 22.0\right.$ $\mathrm{mmol}$, 升至室温后搅拌 $3 \mathrm{~h}$, 反应完毕后直接蒸去溶剂, 用 $\mathrm{CH}_{2} \mathrm{Cl}_{2}$ 萃取 $(100 \mathrm{~mL} \times 3)$, 饱和食盐水洗 $(50 \mathrm{~mL} \times 2)$, 无水 $\mathrm{NaSO}_{4}$ 干燥有机相, 抽滤, 减压浓缩, 硅胶柱层析 $[V($ 石油醚 $): V($ 乙酸乙酯 $)=8: 1$ ] 分离得黄色粘稠状产 品 5, 产率 92\%. $[\alpha]_{\mathrm{D}}^{25}+35.5\left(c 1.0, \mathrm{CHCl}_{3}\right) ;{ }^{1} \mathrm{H} \mathrm{NMR}$ $\left(600 \mathrm{MHz}, \mathrm{CDCl}_{3}\right) \delta: 1.30 \sim 1.50\left(\mathrm{~m}, 15 \mathrm{H}, 5 \mathrm{CH}_{3}\right), 3.34 \sim$ $3.42(\mathrm{~m}, 1 \mathrm{H}, \mathrm{CH}), 3.72 \sim 3.95\left(\mathrm{~m}, 2 \mathrm{H}, \mathrm{CH}_{2}\right), 4.70(\mathrm{dd}, J=$ $12.0,6.0 \mathrm{~Hz}, 1 \mathrm{H}, \mathrm{CH}), 4.70(\mathrm{dd}, J=15.6,6.0 \mathrm{~Hz}, 1 \mathrm{H}, \mathrm{CH})$, 9.62, $9.66(\mathrm{~s}, 1 \mathrm{H}, \mathrm{CHO}) ;{ }^{13} \mathrm{C} \mathrm{NMR}\left(125 \mathrm{MHz}, \mathrm{CDCl}_{3}\right) \delta$ : $24.9,26.8,26.9,28.2,51.7,52.2,58.3,60.3,71.3,71.9$, 78.4, 78.8, 79.4, 79.8, 154.0, 154.5, 197.8, 198.1 (CHO); HRMS (ESI) calcd for $\mathrm{C}_{13} \mathrm{H}_{21} \mathrm{NO}_{5} \mathrm{Na}$ 294.1317, found 294.1329.

3.2 .5 (3aS, $4 R, 6 a R)$-叔丁基-2,2-二甲基-4-(差烡)二 氢-3aH-[1,3] 二氧戊烷 $[4,5-c]$ 吡咯-5(4H)-羒酸酯 $(6)$ 的 合成

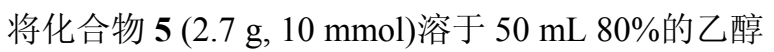

水溶液, 冰浴下滴加硼氢化钠 $\left(\mathrm{NaBH}_{4}, 0.8 \mathrm{~g}, 20 \mathrm{mmol}\right)$ 水溶液, 反应 $1 \mathrm{~h}$ 后滴加饱和氯化铵淬灭, 浓缩, 用 $\mathrm{CH}_{2} \mathrm{Cl}_{2}$ 萃取 $(100 \mathrm{~mL} \times 3)$, 饱和食盐水洗 $(50 \mathrm{~mL} \times 2)$, 无 水 $\mathrm{NaSO}_{4}$ 干燥有机相, 抽滤, 减压浓缩, 硅胶柱层析 $[V($ 石油醚 $): V($ 乙酸乙酯 $)=6: 1$ ] 分离得白色固体 6 , 产 率 94\%. m.p. 98.5 101.3 ${ }^{\circ} \mathrm{C} ;[\alpha]_{\mathrm{D}}^{25}+56.0$ (c 1.0, $\left.\mathrm{CHCl}_{3}\right) ;{ }^{1} \mathrm{H} \mathrm{NMR}\left(600 \mathrm{MHz}, \mathrm{CDCl}_{3}\right) \delta: 1.32\left(\mathrm{~s}, 3 \mathrm{H}, \mathrm{CH}_{3}\right)$, $1.42\left(\mathrm{~s}, 3 \mathrm{H}, \mathrm{CH}_{3}\right), 1.48\left(\mathrm{~s}, 9 \mathrm{H}, 3 \mathrm{CH}_{3}\right), 3.46 \sim 3.53(\mathrm{~m}, 1 \mathrm{H}$, $\mathrm{CH}), 3.63 \sim 3.65\left(\mathrm{~m}, 2 \mathrm{H}, \mathrm{CH}_{2}\right), 3.69(\mathrm{t}, J=11.4 \mathrm{~Hz}, 1 \mathrm{H}$, $\mathrm{CH}), 3.95 \sim 4.00(\mathrm{~m}, 1 \mathrm{H}, \mathrm{CH}), 4.71(\mathrm{t}, J=6.0 \mathrm{~Hz}, 1 \mathrm{H}$, $\mathrm{CH}), 4.77(\mathrm{dd}, J=11.4,6.0 \mathrm{~Hz}, 1 \mathrm{H}, \mathrm{CH}) ;{ }^{13} \mathrm{C}$ NMR $(125$ $\left.\mathrm{MHz}, \mathrm{CDCl}_{3}\right) \delta: 23.6,25.9,27.3,52.5,53.1,60.9,61.3$, 65.0, 65.5, 78.8, 79.5, 79.9, 79.9, 82.1, 82.8, 111.1; HRMS (ESI) calcd for $\mathrm{C}_{13} \mathrm{H}_{23} \mathrm{NO}_{5} \mathrm{Na} 296.1474$, found 296.1482.

3.2.6 (3aS, 4R, 6aR)-叔丁基-2,2-二甲基-4-(叠氮甲基) 二氢- $3 a H-[1,3]$ 二氧戊烷 $[4,5-c]$ 吡咯-5(4H)-羒酸酯 $(8)$ 的合成

将化合物 6 (2.0 g, $9.0 \mathrm{mmol})$ 溶于 $10 \mathrm{~mL}$ 新蒸吡啶溶 液，冰水浴下分批加入对甲苯磺酰氯( $\mathrm{TsCl}, 2.0 \mathrm{~g}, 10.0$ $\mathrm{mmol})$, 待体系稳定后撤去冰水浴, 氮气保护下反应约 2 $\mathrm{h}$, 加冰水淬灭反应, 用稀盐酸中和吡啶, $\mathrm{CH}_{2} \mathrm{Cl}_{2}$ 萃取 $(100 \mathrm{~mL} \times 3)$, 饱和食盐水洗 $(50 \mathrm{~mL} \times 2)$, 无水 $\mathrm{NaSO}_{4}$ 干 燥有机相, 抽滤, 减压浓缩, 硅胶柱层析 $[V$ (石油醚) : $V($ 乙酸乙酯 $)=9 ： 1]$ 分离得白色固体化合物 (3aS, $4 R, 6 a R$ )-叔丁基-2,2-二甲基-4-(对甲苯磺酰氧甲基) 二氢 $-3 a H-[1,3]$ 二氧戊烷 $[4,5-c]$ 吡咯 $-5(4 H)$-羧酸酯 $(7)$, 产率 $92 \%$. 将化合物 $7(3.5 \mathrm{~g}, 8.0 \mathrm{mmol})$ 溶于 $10 \mathrm{~mL}$ $N, N$-二甲基甲酰胺(DMF), 氮气保护下加入叠氮化钠 $\left(\mathrm{NaN}_{3}, 1.1 \mathrm{~g}, 16.0 \mathrm{mmol}\right)$ 并升温至 $100{ }^{\circ} \mathrm{C}$, 反应 $3 \mathrm{~h}$, 待 反应体系冷却至室温后, 水洗 $(50 \mathrm{~mL} \times 5)$, 无水 $\mathrm{NaSO}_{4}$ 干燥有机相, 抽滤, 减压浓缩, 硅胶柱层析 $[V($ 石油 醚) $: V($ 乙酸乙酯 $)=10 ： 1]$ 分离得褐色固体 8 , 产率 94\%. $[\alpha]_{\mathrm{D}}^{25}+38.9$ (c 1.0, $\mathrm{CHCl}_{3}$ ); ${ }^{1} \mathrm{H}$ NMR (600 MHz, $\left.\mathrm{CDCl}_{3}\right) \delta: 1.33\left(\mathrm{~s}, 3 \mathrm{H}, \mathrm{CH}_{3}\right), 1.47\left(\mathrm{~s}, 3 \mathrm{H}, \mathrm{CH}_{3}\right), 1.48(\mathrm{~s}$, $\left.6 \mathrm{H}, 2 \mathrm{CH}_{3}\right), 1.49\left(\mathrm{~s}, 3 \mathrm{H}, \mathrm{CH}_{3}\right), 3.40 \sim 3.52\left(\mathrm{~m}, 2 \mathrm{H}, \mathrm{CH}_{2}\right)$, $3.55 \sim 3.88\left(\mathrm{~m}, 2 \mathrm{H}, \mathrm{CH}_{2}\right), 4.06 \sim 4.16(\mathrm{~m}, 1 \mathrm{H}, \mathrm{CH}), 4.58$ $(\mathrm{d}, J=16.0 \mathrm{~Hz}, 1 \mathrm{H}, \mathrm{CH}), 4.76(\mathrm{~s}, 1 \mathrm{H}, \mathrm{CH}) ;{ }^{13} \mathrm{C} \mathrm{NMR}(125$ $\left.\mathrm{MHz}, \mathrm{CDCl}_{3}\right) \delta:$ 25.0, 27.0, 28.4, 51.8, 52.4, 52.5, 53.1, 62.9, 63.1, 78.6, 79.2, 80.3, 80.4, 82.6, 83.3, 112.0; HRMS (ESI) calcd for $\mathrm{C}_{13} \mathrm{H}_{22} \mathrm{~N}_{4} \mathrm{O}_{4} \mathrm{Na} 321.1538$, found 321.1540 .

\section{3 六元氮杂糖醛基及叠氮基中间体的合成}

$3.3 .1(3 a R, 6 S, 7 R, 7 a S)-2,2-$ 二甲基-6-(三苯甲基氧甲 基)六氢- $[1,3]$ 二氧戊烷 $[4,5-c]$ 吡啶-7-醇(10)的合成

参考文献 $[20,21]$ 的方法，合成中间体化合物 (3aS,6R,6aS)-2,2-二甲基-6-[( $S)$-1-叠氮基-2-(三苯甲基氧 
基)乙基]四氢呋喃 [3,4- $d][1,3]$ 二氧戊烷-4-醇(9). 将化合 物 9 (20.0 g, $41.0 \mathrm{mmol}$ ) 溶于 $150 \mathrm{~mL}$ 四氢呋喃(THF), 加 入 $\mathrm{PPh}_{3} 14.0 \mathrm{~g}, \mathrm{~N}_{2}$ 保护下 $70{ }^{\circ} \mathrm{C}$ 回流 $1 \mathrm{~h}$, 蒸去 THF, 加 甲醇溶解，冰浴下分批缓慢加入 $\mathrm{NaBH}_{4}(4.7 \mathrm{~g}, 123.0$ $\mathrm{mmol}$ ), 反应过夜, 加饱和 $\mathrm{NH}_{4} \mathrm{Cl}$ 水溶液淬灭, 蒸去甲 醇, 用 $\mathrm{CH}_{2} \mathrm{Cl}_{2}$ 萃取 $(200 \mathrm{~mL} \times 3)$, 无水 $\mathrm{Na}_{2} \mathrm{SO}_{4}$ 干燥有机 相, 抽滤, 减压浓缩, 硅胶柱层析 $[V$ (石油醚) $: V$ (乙酸乙 酯) $=10: 1$ ]分离得白色固体 10, 产率 $85 \%$. m.p. 98.5 $101.3{ }^{\circ} \mathrm{C} ;[\alpha]_{\mathrm{D}}^{25}+3.0\left(c 1.0, \mathrm{CHCl}_{3}\right) ;{ }^{1} \mathrm{H} \mathrm{NMR}(600 \mathrm{MHz}$, $\left.\mathrm{CDCl}_{3}\right) \delta: 1.37\left(\mathrm{~s}, 3 \mathrm{H}, \mathrm{CH}_{3}\right), 1.53\left(\mathrm{~s}, 3 \mathrm{H}, \mathrm{CH}_{3}\right), 2.74(\mathrm{dd}$, $J=12.0,5.4 \mathrm{~Hz}, 1 \mathrm{H}, \mathrm{CH}), 3.10 \sim 3.24\left(\mathrm{~m}, 2 \mathrm{H}, \mathrm{CH}_{2}\right)$, $3.38 \sim 3.42\left(\mathrm{~m}, 2 \mathrm{H}, \mathrm{CH}_{2}\right), 3.92(\mathrm{~s}, 1 \mathrm{H}, \mathrm{CH}), 4.20(\mathrm{q}, J=$ $2.4 \mathrm{~Hz}, 1 \mathrm{H}, \mathrm{CH}), 4.30(\mathrm{dd}, J=14.4,6.0 \mathrm{~Hz}, 1 \mathrm{H}, \mathrm{CH})$, $7.45 \sim 7.26$ (m, $15 \mathrm{H}, \mathrm{ArH}) ;{ }^{13} \mathrm{C} \mathrm{NMR}\left(125 \mathrm{MHz}, \mathrm{CDCl}_{3}\right)$ $\delta: 26.1,28.1,47.6,55.0,64.2,67.2,70.4,76.0,87.2,109.2$, 127.2, 128.0, 128.6, 143.5; HRMS (ESI) calcd for $\mathrm{C}_{28} \mathrm{H}_{31}$ $\mathrm{NO}_{4} \mathrm{Na} 468.2151$, found 468.2139.

3.3.2 (3aR, $6 S, 7 R, 7 a S$ ) - 叔丁基-2,2-二甲基-6-(羟甲 基)-7-(苯甲酰氧基)四氢- $[1,3]$ 二氧戊烷 $[4,5-c]$ 吡啶$5(6 H)$ - 羒酸酯 $(13)$ 的合成

用合成化合物 $\mathbf{4}$ 的方法合成化合物 $(3 a R, 6 S, 7 R, 7 a S)$ 叔丁基-2,2-二甲基-6-(三苯甲基氧甲基)-7-(羟基)四氢$[1,3]$ 二氧戊烷 $[4,5-c]$ 吡啶-5(6H)-羧酸酯(11). 将化合物 11 (3.0 g, $5.4 \mathrm{mmol})$ 溶于 $40 \mathrm{~mL}$ 新蒸吡啶, 冰浴下缓慢 滴入苯甲酰氯( $\mathrm{BzCl}, 1.0 \mathrm{~mL} 8.1 \mathrm{mmol}), \mathrm{N}_{2}$ 保护下室温 摚拌 $4 \mathrm{~h}$, 加水淬灭, 稀盐酸中和吡啶, 用 $\mathrm{CH}_{2} \mathrm{Cl}_{2}$ 萃取 $(200 \mathrm{~mL} \times 3)$, 无水 $\mathrm{Na}_{2} \mathrm{SO}_{4}$ 干燥有机相, 抽滤, 减压浓 缩, 硅胶柱层析 $[V$ (石油醚) $: V($ 乙酸乙酯 $)=6: 1]$ 分离 得白色固体化合物(3aR, $6 S, 7 R, 7 a S)$-叔丁基-2,2-二甲基6-(三苯甲基氧甲基)-7-(苯甲酰氧基)四氢-[1,3]二氧戊烷 [4,5-c]吡定-5(6H)-羧酸酯(12), 产率 $90 \%$. 将化合物 $\mathbf{1 2}$

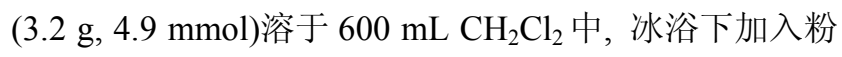
末状 $\mathrm{FeCl}_{3} \cdot 6 \mathrm{H}_{2} \mathrm{O}(2.0 \mathrm{~g}, 7.4 \mathrm{mmol})$, 搅拌约 $20 \mathrm{~min}$ 后加 水淬灭, 水洗 $(80 \mathrm{~mL} \times 3)$, 用无水 $\mathrm{Na}_{2} \mathrm{SO}_{4}$ 干燥有机相, 抽滤, 减压浓缩, 硅胶柱层析 [ $V$ (石油醚) : $V($ 乙酸乙 酯 $)=12:$ ] 分离得棕黄色油状物 $\mathbf{1 3}$ 和原料 $\mathbf{1 2}$.

化合物 13: 产率为 $72 \%,[\alpha]_{\mathrm{D}}^{25}+26.3$ (c 1.0 , $\left.\mathrm{CHCl}_{3}\right) ;{ }^{1} \mathrm{H} \mathrm{NMR}\left(600 \mathrm{MHz}, \mathrm{CDCl}_{3}\right) \delta: 1.35\left(\mathrm{~s}, 3 \mathrm{H}, \mathrm{CH}_{3}\right)$, $1.46 \sim 1.52\left(\mathrm{~m}, 12 \mathrm{H}, 4 \mathrm{CH}_{3}\right), 3.33 \sim 3.71\left(\mathrm{~m}, 2 \mathrm{H}, \mathrm{CH}_{2}\right)$, $3.87 \sim 4.96(\mathrm{~m}, 1 \mathrm{H}, \mathrm{CH}), 4.21 \sim 4.53\left(\mathrm{~m}, 4 \mathrm{H}, \mathrm{CH}_{2}, 2 \mathrm{CH}\right)$, $5.73(\mathrm{t}, J=4.2 \mathrm{~Hz}, 1 \mathrm{H}, \mathrm{CH}), 7.48 \sim 8.11(\mathrm{~m}, 5 \mathrm{H}, \mathrm{ArH}) ;{ }^{13} \mathrm{C}$ NMR $\left(125 \mathrm{MHz}, \mathrm{CDCl}_{3}\right) \delta$ : 24.3, 26.3, 28.3, 28.4, 43.0, 53.6, 60.4, 63.1, 68.7, 71.3, 72.3, 80.9, 110.1, 157.8, 1654, 171.2; HRMS (ESI) calcd for $\mathrm{C}_{21} \mathrm{H}_{30} \mathrm{NO}_{7} 408.2022$, found 408.2008.
3.3.3（3aR, 6S,7R,7aS)-叔丁基-2,2-二甲基-6-甲酰基7-(苯甲酰氧基)四氢- $[1,3]$ 二氧戊烷 $[4,5-c]$ 吡啶-5(6H)羧酸酯(14)的合成

将化合物 $\mathbf{1 3}$ (1.8 g, $4.4 \mathrm{mmol}$ )溶于 $40 \mathrm{~mL} \mathrm{CH}_{2} \mathrm{Cl}_{2}$ 中, 加入戴斯马丁试剂(Dess-Martin 试剂, $2.0 \mathrm{~g}, 4.8 \mathrm{mmol}$ ), $\mathrm{N}_{2}$ 保护下室温搅拌 $1 \mathrm{~h}$, 加入少量 $\mathrm{K}_{2} \mathrm{CO}_{3}$ 淬灭, 抽滤, 减 压浓缩, 硅胶柱层析 $[($ 石油醚 $): V($ 乙酸乙酯 $)=8: 1]$ 分离得浅黄色油状物 14, 产率为 $90 \%$. $[\alpha]_{\mathrm{D}}^{25}+51.7(c$ 1.0, $\left.\mathrm{CHCl}_{3}\right) ;{ }^{1} \mathrm{H}$ NMR $\left(600 \mathrm{MHz}, \mathrm{CDCl}_{3}\right) \delta: 1.33 \sim 1.51$ $\left(\mathrm{m}, 15 \mathrm{H}, 5 \mathrm{CH}_{3}\right), 3.45 \sim 3.55(\mathrm{~m}, 1 \mathrm{H}, \mathrm{CH}), 4.33 \sim 4.71(\mathrm{~m}$, $\left.4 \mathrm{H}, \mathrm{CH}_{2}, 2 \mathrm{CH}\right), 5.76 \sim 5.87(\mathrm{~m}, 1 \mathrm{H}, \mathrm{CH}), 7.40 \sim 8.00(\mathrm{~m}$, $5 \mathrm{H}, \mathrm{ArH}), 9.55 \sim 9.61(\mathrm{~m}, 1 \mathrm{H}, \mathrm{CHO}) ;{ }^{13} \mathrm{C}$ NMR $(125$ $\left.\mathrm{MHz}, \mathrm{CDCl}_{3}\right) \delta: 24.1,24.2,26.3,26.4,28.2,28.2,41.2$, $42.6,59.7,59.9,68.2,68.9,70.6,70.7,72.1,72.2,81.2$, $81.6,110.1,110.2,128.6,128.7,129.6,129.7,133.8$, $133.9,155.3,156.2,164.7,164.7,198.5,199.3$; HRMS (ESI) calcd for $\mathrm{C}_{21} \mathrm{H}_{27} \mathrm{NO}_{7} \mathrm{Na} 428.1685$, found 428.1682 .

3.3.4 2,2,2-三氟基-1-[(3aR, $6 S, 7 R, 7 a S)-2,2$-二甲基6 -(三苯甲基氧甲基)-7-(羟基)四氢-[1,3] 二氧戊烷 $[4,5-c]$ 吡啶-5(6H)-基]乙基酮(15)的合成

将化合物 10 (12.0 g, $27.0 \mathrm{mmol}$ )溶于 $200 \mathrm{~mL}$ 新蒸 $\mathrm{CH}_{2} \mathrm{Cl}_{2}$, 加入吡啶 $40 \mathrm{~mL}$, 在 $-40{ }^{\circ} \mathrm{C}$ 下滴加三氟乙酸䣶 (TFAA) $12.6 \mathrm{~mL}(80.9 \mathrm{mmol}), \mathrm{N}_{2}$ 保护下反应约 $1 \mathrm{~h}$ 后, 加水淬灭, 用稀盐酸中和吡啶, 用 $\mathrm{CH}_{2} \mathrm{Cl}_{2}$ 萃取 (200 $\mathrm{mL} \times 3)$, 无水 $\mathrm{Na}_{2} \mathrm{SO}_{4}$ 干燥有机相, 抽滤, 减压浓缩, 硅 胶柱层析 $[V$ (石油醚) $: V$ (乙酸乙酯 $)=10 ： 1]$ 分离得黄色 油状黏状物 15 , 产率为 $80 \%$. $[\alpha]_{\mathrm{D}}^{25}+18.0$ (c 1.0 , $\left.\mathrm{CHCl}_{3}\right) ;{ }^{1} \mathrm{H} \mathrm{NMR}\left(600 \mathrm{MHz}, \mathrm{CDCl}_{3}\right) \delta: 1.32\left(\mathrm{~s}, 3 \mathrm{H}, \mathrm{CH}_{3}\right)$, 1.34 (s, $\left.3 \mathrm{H}, \mathrm{CH}_{3}\right), 3.45 \sim 3.55\left(\mathrm{~m}, 2 \mathrm{H}, \mathrm{CH}_{2}\right), 3.59(\mathrm{~s}, 1 \mathrm{H}$, $\mathrm{CH}), 3.89 \sim 3.92\left(\mathrm{~m}, 2 \mathrm{H}, \mathrm{CH}_{2}\right), 4.38 \sim 4.44(\mathrm{~m}, 3 \mathrm{H}, 3 \mathrm{CH})$, $7.23 \sim 7.39$ (m, $15 \mathrm{H}, \mathrm{ArH}) ;{ }^{13} \mathrm{C}$ NMR $\left(125 \mathrm{MHz}, \mathrm{CDCl}_{3}\right)$ $\delta: 24.1,26.1,44.2,52.1,61.5,67.4,71.8,72.5,87.8,110.0$, 127.5, 128.2, 128.2; HRMS (ESI) calcd for $\mathrm{C}_{30} \mathrm{H}_{30} \mathrm{~F}_{3} \mathrm{NO}_{5}$ Na 564.1973, found 564.1966.

3.3.5 (3aR, $6 R, 7 R, 7 a R)-2,2$-二甲基-6-(叠氮甲基)-5$(2,2,2$-三氟乙酰基)-[1,3] 二氧戊烷 $[4,5-c]$ 六氢吡啶-7苯甲酸酯(19)的合成

用合成化合物 12 和 13 的方法来合成化合物( $3 a R$, $6 R, 7 R, 7 a R$ )-2,2-二甲基-6-(三苯甲基氧甲基)-5-(2,2,2-三 氟乙酰基)六氢- $[1,3]$ 二氧戊烷 $[4,5-c]$ 吡啶-7-苯甲酸酯 (16)和( $3 a R, 6 R, 7 R, 7 a R$ )-2,2-二甲基-6-(羟甲基)-5-(2,2,2三氟乙酰基)六氢- $[1,3]$ 二氧戊烷 $[4,5-c]$ 吡啶-7-苯甲酸酯 (17), 然后将化合物 17 (7.1 g, $17.6 \mathrm{mmol}$ )溶于新蒸 $\mathrm{CH}_{2} \mathrm{Cl}_{2}$ 中, 加入 $\mathrm{Et}_{3} \mathrm{~N} 80 \mathrm{~mL}$ 和 DMAP $(0.3 \mathrm{~g}, 2.1 \mathrm{mmol})$, 冰浴下加入 $\mathrm{TsCl}(6.7 \mathrm{~g}, 35.0 \mathrm{mmol})$, 反应 $1 \mathrm{~h}$ 后加水淬 
灭, 稀盐酸中和, $\mathrm{CH}_{2} \mathrm{Cl}_{2}$ 萃取 $(200 \mathrm{~mL} \times 3)$, 无水 $\mathrm{Na}_{2} \mathrm{SO}_{4}$ 干燥有机相, 抽滤, 减压浓缩, 硅胶柱层析 [ $V$ (石油 醚) $: V$ (乙酸乙酯 $)=12: 1$ 分离得浅黄色固体, 产率为 $90 \%$. 将化合物( $3 a R, 6 R, 7 R, 7 a R$ )-2,2-二甲基-6-对甲苯磺 酰氧基甲基-5-(2,2,2-三氟乙酰基)六氢-[1,3]二氧戊烷 [4,5-c]吡啶-7-苯甲酸酯 $(\mathbf{1 8})(9.8 \mathrm{~g}, 17.6 \mathrm{mmol})$ 溶于新蒸 的 DMF 中, 加入 $\mathrm{NaN}_{3}(3.4 \mathrm{~g}, 52.8 \mathrm{mmol}), \mathrm{N}_{2}$ 保护下 100 ${ }^{\circ} \mathrm{C}$ 搅拌 $5 \mathrm{~h}$, 待体系冷却至室温后水洗 $(50 \mathrm{~mL} \times 3)$, 无水 $\mathrm{Na}_{2} \mathrm{SO}_{4}$ 干燥, 抽滤, 减压浓缩, 硅胶柱层析 [ $V$ (石油 醚)：V(乙酸乙酯 $)=10: 1$ ]分离得白色固体 19 , 产率为 $75 \%$. m.p. $124.0 \sim 125.0{ }^{\circ} \mathrm{C}$; $[\alpha]_{\mathrm{D}}^{25}+49.2\left(\right.$ c $\left.1.0, \mathrm{CHCl}_{3}\right)$; ${ }^{1} \mathrm{H}$ NMR $\left(600 \mathrm{MHz}, \mathrm{CDCl}_{3}\right) \delta: 1.35\left(\mathrm{~s}, 3 \mathrm{H}, \mathrm{CH}_{3}\right), 1.42$ (s, $\left.3 \mathrm{H}, \mathrm{CH}_{3}\right), 3.93\left(\mathrm{~d}, J=15.0 \mathrm{~Hz}, 2 \mathrm{H}, \mathrm{CH}_{2}\right), 4.44$ (d, $J=7.8$ $\mathrm{Hz}, 1 \mathrm{H}, \mathrm{CH}), 4.59$ (q, $J=3.6 \mathrm{~Hz}, 1 \mathrm{H}, \mathrm{CH}), 4.75 \sim 4.82(\mathrm{~m}$, $1 \mathrm{H}, \mathrm{CH}), 5.84$ (t, $J=4.2 \mathrm{~Hz}, 1 \mathrm{H}, \mathrm{CH}), 7.52 \sim 8.04(5 \mathrm{H}, \mathrm{m}$, $\mathrm{ArH}) ;{ }^{13} \mathrm{C}$ NMR $\left(125 \mathrm{MHz}, \mathrm{CDCl}_{3}\right) \delta: 24.1,25.9,44.4$, 47.6, 51.2, 67.0, 70.5, 71.4, 110.8, 128.8, 129.7, 133.9, 158.0, 164.9; HRMS (ESI) calcd for $\mathrm{C}_{18} \mathrm{H}_{19} \mathrm{~F}_{3} \mathrm{~N}_{4} \mathrm{O}_{5} \mathrm{Na}$ 451.1205 , found 451.1212 .

\section{References}

[1] Horne, G.; Wilson, F. X.; Tinsley, J.; David, H. W.; Richard, S. J. Drug Discovery Today 2011, 16, 107.

[2] Compain, P.; Martin, O. R. Iminosugars: From Synthesis to Therapeutic Applications, John Wiley \& Sons Ltd, England, 2007.

[3] Stütz; Arnold, E. Iminosugars as Glycosidase Inhibitors: Nojirimycin and Beyond, Wiley-VCH, Weinheim, 1999.

[4] Winchester, B. G. Tetrahedron: Asymmetry 2009, 20, 645.

[5] Wrodnigg, T. M.; Steiner, A. J.; Ueberbacher, B. J. Anti-Cancer Agents Med. Chem. 2008, 8, 77.

[6] Natoria, Y.; Imahoria, T.; Murakamia, K.; Yoshimuraa, Y.; Nakagawab, S.; Katob, A.; Adachib, I.; Takahata, H. Bioorg. Med. Chem. Lett. 2011, 21, 738.
[7] Diot, J. D.; Moreno, I. G.; Twigg, G.; Mellet, C. O.; Haupt, K.; Butters, T. D.; Kovensky, J.; Gouin, S. G. J. Org. Chem., 2011, 76, 7757.

[8] Clinch, K.; Evans, G. B.; Frohlich, R. F. G.; Furneaux, R. H.; Kelly, P. M.; Legentil, L.; Murkin, A. S.; Li, L. Schramm, V. L.; Tyler, P. C.; Woolhouse, A. D. J. Med. Chem. 2009, 52, 1126.

[9] de Melo, E. B.; da Silveira, G. A.; Carvalho, I. Tetrahedron 2006, 62, 10277.

[10] Wrodnigg, T. M.; Diness, F.; Gruber, C.; Hä usler, H.; Lundt, I.; Rupitz, K.; Steiner, A. J.; Stütz, A. E.; Tarling, C. A.; Withers, S. G.; Wölfler, H. Bioorg. Med. Chem. 2004, 12, 3485.

[11] Ikeda, K.; Takahashi, M.; Nishida, M.; Miyauchi, M.; Kizu, H.; Kameda, Y.; Arisawa, M.; Watson, A. A.; Nash, R. J.; Fleet, G. W. J.; Asano, N. Carbohydr. Res. 2000, 323, 73.

[12] Afarinkia, K.; Bahar, A. Tetrahedron: Asymmetry 2005, 16, 1239.

[13] Chen, H.; Yin, Q. M.; Li, C. X.; Wang, E. K.; Gao, F.; Zhang, X. B.; Yin, Z.; Wei, S. N.; Li, X. L.; Meng, M.; Zhang, P. Z.; Li, N.; Zhang, J. C. ACS Med. Chem. Lett. 2011, $2,845$.

[14] Li, X. L.; Yin, Q. M.; Jiao, L. L.; Qin, Z. B.; Feng, J. N.; Chen, H.; Zhang, J. C.; Meng, M. Carbohydr. Res. 2011, 346, 401.

[15] Ye, X. S.; Sun, F.; Liu, M.; Li, Q.; Wang, Y. H.; Zhang, G. S.; Zhang, L. H.; Zhang, X. L. J. Med. Chem. 2005, 48, 3688.

[16] Li, X. L.; Zhang, P. Z.; Tian, J.; Duan, K. F.; Chen, H. Chin. J. Org. Chem. 2007, 27, 1013 (in Chinese).

(李小六, 张平竹, 田军, 段科芳, 陈华, 有机化学, 2007, 27, 1013.)

[17] Li, X. L.; Qin, Z. B.; Wang, R.; Chen, H.; Zhang, P. Z. Tetrahedron 2011, 67, 1792.

[18] Fleet, G. W. J.; Sona, J. C.; Greenb, D. S. C.; Bellob, I. C.; Winchesterb, B. Tetrahedron 1988, 44, 2649.

[19] Chang, Y. C.; Chir, J. L.; Tsai, S. Y.; Juang, W. F.; Wu, A. T. Tetrahedron Lett. 2009, 50, 4925.

[20] Ménand, M.; Blais, J. C. Valéry, J. M.; Xie, J. J. Org. Chem., 2006, $71,3295$.

[21] Moon, H. R.; Choi, W. J.; Kim, H. O.; Jeong. L. S. Tetrahedron: Asymmetry 2002, 13, 1189.

[22] Davis, B. G. Tetrahedron: Asymmetry 2009, 20, 652.

[23] Curran, T. P.; Pollastri, M. P.; Abelleira, S. M.; Messier, R. J.; Mccollum, T. A.; Rowe, C. G. Tetrahedron Lett. 1994, 35, 5409.

[24] Fleet, G. W. J.; Smith, P. W. Tetrahedron 1987, 47, 979.

(Li, L.; Fan, Y.) 